\title{
Reduction of Speckles and Distortion in Projection System by Using a Rotating Diffuser
}

\author{
Chien-Yue Chen ${ }^{1}$, Wei-Chia Su${ }^{2}$, Ching-Huang Lin $^{1}$, Ming-De $\mathrm{KE}^{3}$, Qing-Long Deng ${ }^{4}$, and Kuan-Yao Chiu ${ }^{1}$ \\ ${ }^{1}$ Department of Electronic Engineering, National Yunlin University of Science and Technology, Douliou, Yunlin 64002, Taiwan \\ ${ }^{2}$ Graduate Institute of Photonics, National Changhua University of Education, Changhua 50007, Taiwan \\ ${ }^{3}$ Graduate School of Engineering Science and Technology, National Yunlin University of Science and Technology, \\ Douliou, Yunlin 64002, Taiwan \\ ${ }^{4}$ Institute of Photonic Systems, National Chiao Tung University, Guiren, Tainan 71150, Taiwan
}

(Received May 31, 2012; Accepted August 31, 2012)

\begin{abstract}
A head up display (HUD) with laser backlight unit has two principle problems; one is laser speckles and the other is distortion. In this study, we propose that a rotating diffuser set at the intermediate image plane of the HUD projection system can simultaneously solve these problems. The conditions of speckle reduction and de-distortion are also estimated in this paper. (C) 2012 The Japan Society of Applied Physics
\end{abstract}

Keywords: head-up display (HUD), laser speckle, distortion

\section{Introduction}

A car-used head up display (HUD) is used for reducing accidents caused by drivers being tired for a long period of time and losing the concentration. As general HUD use LED as the backlight and appear the problems of low resolution, small projection area, short projection distance, and view obstruction, laser backlight is introduced. The speckle interference in laser projection systems had been studied since 1965, and several solutions were proposed with nearfield optics and far-field optics. Wang et al. proposed to reduce speckles with diffractive optical element (DOE) in near-field optics that the speckle contrast was dropped down to $3.8 \%$. $^{1)}$ Rotating light guide could reduce the speckle contrast up to $1.5 \%$ as well; however, the structure was rather complex and the expense was higher. ${ }^{2)}$ The most common method at present is fast scanning micromirror, ${ }^{3)}$ which replaces rotations to reduce speckles. Nevertheless, the structure is also complicated. In far-field optics, the size of speckles enlarges with the increase of projection distance resulting in discomfort when viewing images. ${ }^{4)}$ As a consequence, this study proposes to destroy laser speckles in far-field optics and reduce image distortion with a rotating diffuser by using laser speckles resulted from having laser as the backlight and aberration generated by projection. In this case, the reduction of speckle distribution on laser head up displays and the improvement of projecting quality could facilitate the feasibility of applying laser backlight to medium- and small-size panels.

\section{Principle of Use Diffuser}

\subsection{Speckle reduction}

In the experiment of reducing laser speckles, a diffuser was utilized for improving the image system. When laser passed through the surface, the randomly distributed surface would destroy the wavefront of laser and high coherency, forming uniform scattering light. For phases, the initial phase would become random phase distribution after passing the diffuser. Besides, with the characteristics of rotating diffusers, laser

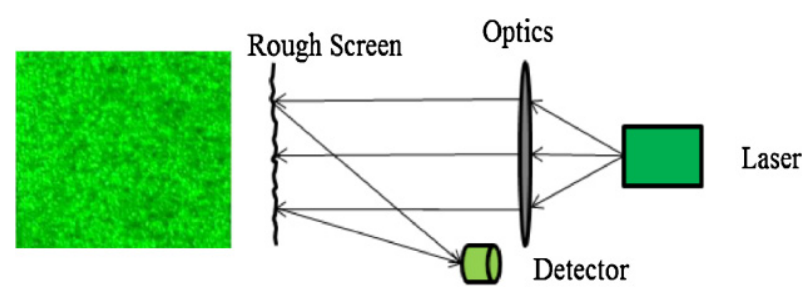

Fig. 1. (Color online) Speckle formation.

would appear uniformly but partly non-coherent light that the speckles would be reduced. Equation (1) is defined as the speckle contrast,${ }^{5)}$ with the range between $0-1$ :

$$
C=\frac{\sqrt{\left\langle I^{2}\right\rangle-\langle I\rangle^{2}}}{\langle I\rangle},
$$

where $C$ stands for the speckle contrast and $I$ is the light intensity. With the high coherency of laser, the light was reflected to detector (human eyes) through the rough surface, where the light intensity loss would increase with the projection distance and light diffusion ${ }^{6}$ (Fig. 1). Nonetheless, the use of diffusers could reduce the speckle contrast, but also result in the loss of laser intensity.

\subsection{Aberration reduction}

In regard to the projecting image quality of head up displays, pincushion distortion is likely to appear, as the image position is larger than the ideal image position, when off-axis (Fig. 2). $\overline{y_{\mathrm{I}}}$ represents the object being imaged on the ideal position, and $\overline{y_{\mathrm{R}}}$ stands for the real image position. A diffuser was further utilized for reducing the image distortion $^{7}$ in this study that the diffuser was placed on the intermediate image plane to correct the lateral magnification when the distance between image and optical axis was different as

$$
\text { distortion rate }(\%)=\left(\frac{\overline{y_{\mathrm{R}}}-\overline{y_{\mathrm{I}}}}{\overline{y_{\mathrm{I}}}}\right) \times 100,
$$




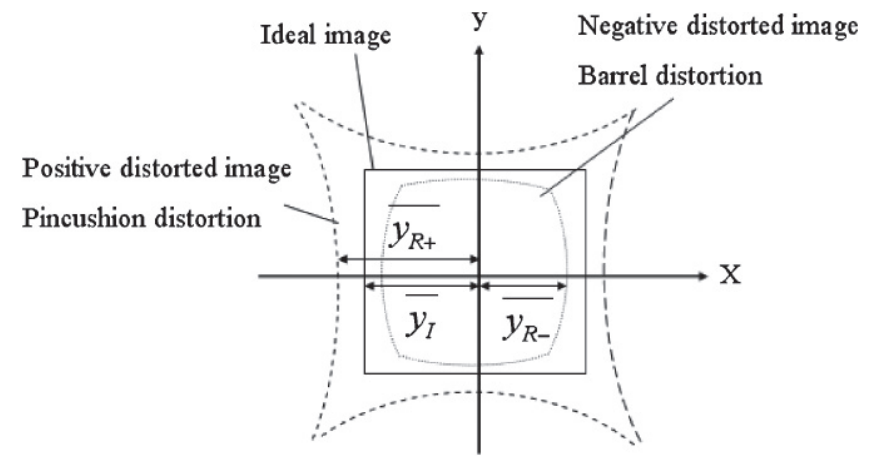

Table 1. Specifications of lens.

\begin{tabular}{ccccc}
\hline Lens & $\begin{array}{c}\text { Focal length } \\
(\mathrm{mm})\end{array}$ & $\begin{array}{c}\text { Curvature } \\
(\mathrm{mm})\end{array}$ & $\begin{array}{c}\text { Thickness } \\
(\mathrm{mm})\end{array}$ & $\begin{array}{c}\text { Diameter } \\
(\mathrm{mm})\end{array}$ \\
\hline 1 & 150.0 & 155.7 & 7.1 & 50.0 \\
2 & 60.0 & 59.3 & 14.4 & 50.8 \\
3 & 301.8 & 311.4 & 11.1 & 100.0 \\
4 & 501.3 & 519.0 & 7.8 & 100.0 \\
\hline
\end{tabular}

Fig. 2. Distortion diagram.

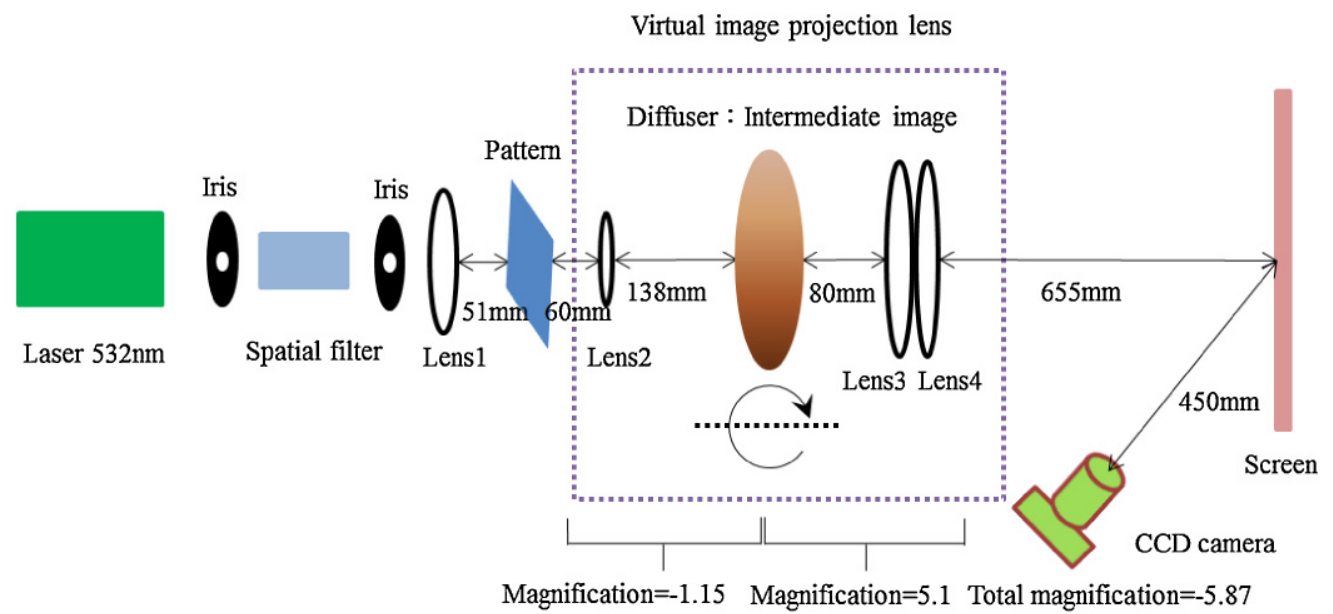

Fig. 3. (Color online) Speckle elimination with rotating diffuser.

\section{Optical Design}

The experimental design of a virtual image projection system with VIS DPSS laser, wavelength $532 \mathrm{~nm}$ and power $50 \mathrm{~mW}$, is shown in Fig. 3. A diffuser [size $50 \times 50 \mathrm{~mm}^{2}$, surface roughness average (RA) $1.57 \mu \mathrm{m}$ ], which was made by grinding $\mathrm{Al}_{2} \mathrm{O}_{3}$ powder, was placed on the intermediate image plane to reduce laser speckles and correct image distortion. The specifications of lens in the system are listed in Table 1.

The spatial filter was used for filtering clutter and expanding beams; Iris was used for controlling the size of beams; Lens1 transformed the expanded beam into collimated light, passing through the Pattern of National Yunlin University of Science and Technology; and the virtual image projection system was placed at the back. In this system, Lens2 generated a real image with the magnification of 1.15 on the intermediate image plane, and Lens3 and Lens4 received a vertical virtual image with the magnification of 5.10 to be projected to the screen. The final image was a virtual image with the magnification of 5.87 at $655 \mathrm{~mm}$ away from Lens4. The material of all lenses was BK7, Lens3 and Lens4 were a set of compound lenses.

The intensity was measured on the screen by CCD camera witch distanced $450 \mathrm{~mm}$ from the screen, with the focus $37 \mathrm{~mm}$, clear aperture $2.8 \mathrm{~mm}$, exposure duration $1 / 50 \mathrm{~s}$, and ISO 200. Then, the speckle contrast was calculated by eq. (1). As speckles would change with the exposure duration and shooting distance, a fixed distance was required for quantitative shooting. ${ }^{8)}$

\section{Experimental Results}

\subsection{Measurement of speckle data}

The experimental structure is shown in Fig. 1. Pattern, originally sized $13 \times 13 \mathrm{~mm}^{2}$, was magnified, through Lens2, a real image on the diffuser at the intermediate image plane sized $15 \times 15 \mathrm{~mm}^{2}$ and further magnified, through the virtual image projection system, a virtual image on the screen sized $76 \times 76 \mathrm{~mm}^{2}$. VIS DPSS Laser with the wavelength $532 \mathrm{~nm}$ was utilized, and the fixed speed for the rotating diffuser was $2 \pi \mathrm{rad} / \mathrm{s}$. Figure 4 shows the virtual image shot by the CCD camera, without a diffuser, and with the speckle contrast $58.8 \%$ [Fig. 4(a)], with a diffuser and the speckle contrast $22.4 \%$ [Fig. 4(b)], and with a rotating diffuser and the speckle contrast 3.1\% [Fig. 4(c)]. Apparently, the speckle contrast would be reduced to a certain degree when laser passing through a rotating diffuser; and, human eyes would not be able to distinguish the image when the contrast was lower than 4.0\%. ${ }^{9}$ In terms of energy dissipation, a power meter was utilized for measuring the loss of light intensity before and after passing through the diffuser. When the light passing the diffuser, the light 


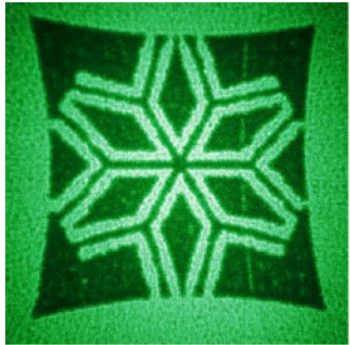

(a)

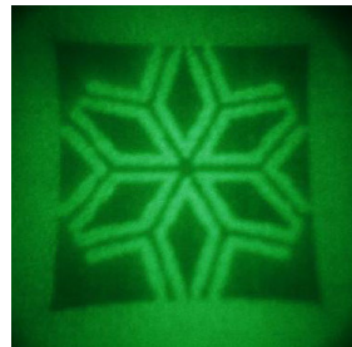

(b)

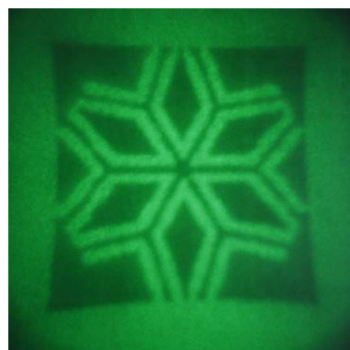

(c)

Fig. 4. (Color online) Speckle patterns from a VIS DPSS laser: (a) without using a diffuser, (b) with a static diffuser, and (c) with a diffuser rotating at a speed of $2 \pi \mathrm{rad} / \mathrm{s}$.

Table 2. Experimental results.

\begin{tabular}{ccccc}
\hline Figure & $\begin{array}{c}\overline{y_{\mathrm{R}}} \\
(\mathrm{mm})\end{array}$ & $\begin{array}{c}\overline{y_{\mathrm{I}}} \\
(\mathrm{mm})\end{array}$ & $\begin{array}{c}\text { Distortion } \\
\text { rate } \\
(\%)\end{array}$ & $\begin{array}{c}\text { Speckle } \\
\text { contrast } \\
(\%)\end{array}$ \\
\hline $5(\mathrm{a})$ & 44.0 & 38.0 & 15.79 & 58.8 \\
$5(\mathrm{~b})$ & 40.5 & 38.0 & 6.58 & 22.4 \\
$5(\mathrm{c})$ & 40.5 & 38.0 & 6.58 & 3.1 \\
\hline
\end{tabular}

intensity was dissipated $62.5 \%$ (energy efficiency) because of the light intensity uniformization. In this case, a diffuser could largely reduce the light intensity. According to Goodman, the irregular geometric figures on the diffuser surface would cause laser scattering, where the larger scattering angle appeared, the more light intensity was dissipated. However, it actually achieved less than $4.0 \%$ speckle contrast. ${ }^{10)}$

\subsection{Measurement of distortion rate}

According to Fig. 2, the magnification of the original image was $5.87,76 \times 76 \mathrm{~mm}^{2}$. The distortion rate was calculated with eq. (2), Table 2. Figure 5(a) shows the virtual image with pincushion distortion, in which $\overline{y_{\mathrm{R}}}$ was $44.0 \mathrm{~mm}, \overline{y_{\mathrm{I}}} 38.0 \mathrm{~mm}$, and the distortion rate $15.79 \%$, while $\overline{y_{\mathrm{R}}}$ was $40.5 \mathrm{~mm}, \overline{y_{\mathrm{I}}} 38.0 \mathrm{~mm}$, and the distortion rate $6.58 \%$ in Fig. 5(b). The diffuser was utilized for correcting the distortion rate of the projection system.

\section{Discussion}

\subsection{Speckle contrast}

Laser speckle contrast would change with the rotating speed and diffuser structure. A diffuser with the RA $1.57 \mu \mathrm{m}$

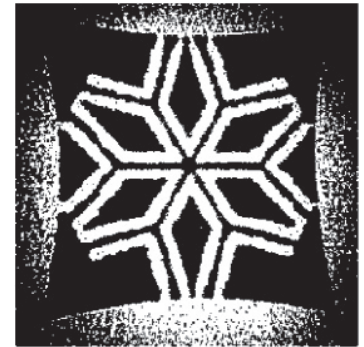

(a)

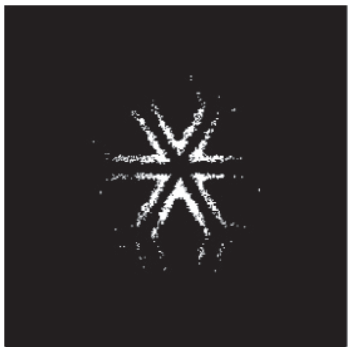

(b)

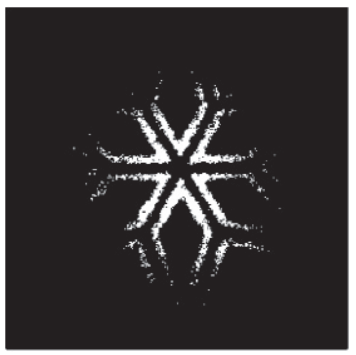

(c)

Fig. 5. Image processing of binary image: (a) without using a diffuser, (b) with a static diffuser, and (c) with a diffuser rotating at a speed of $2 \pi \mathrm{rad} / \mathrm{s}$.

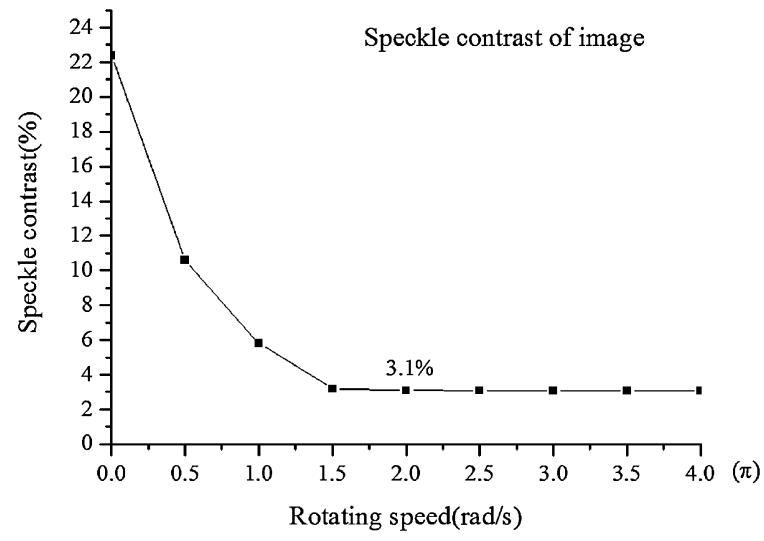

Fig. 6. Speckle contrast of images.

and the rotating speed $0-4 \pi \mathrm{rad} / \mathrm{s}$ was utilized for this experiment, Fig. 6 . When the rotating speed was larger than $2 \pi$, the speckle contrast did not appear large difference. The contrast was measured the contrast of the measured scattering speckle distribution and light intensity by a CCD camera from the fixed distance $450 \mathrm{~mm}$. The shot image was processed with MATLAB. The reduction of binarized speckle intensity and contrast could be clearly seen in Fig. 5. Figure 5(a) shows the speckle distribution of light intensity without a diffuser; Fig. 5(b) displays the 62.5\% loss of light intensity with a diffuser; and Fig. 5(c) presents the light intensity distribution with a rotating diffuser. In comparison with Fig. 5(b), the light intensity was uniformly distributed on the screen and the contrast was dropped down to $3.1 \%$ because of uniformization. 


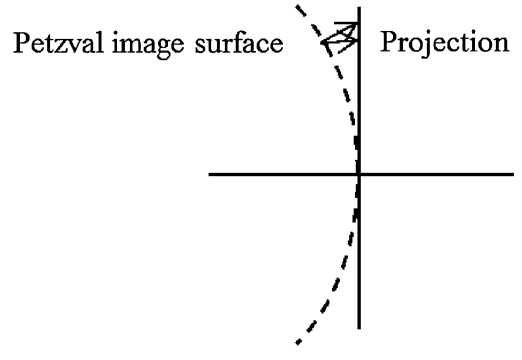

Diffuser screen: Ideal intermediate image plane

Fig. 7. The intermediate image in an imaging system with a diffuser is the projection of the Petzval image on the diffuser screen.

\subsection{Distortion aberration}

Two factors were discovered in diffuser reducing distortion, including (1) Petzval image appearing on the diffuser surface when projecting, and (2) the diffusion effect of the diffuser. Figure 7 shows that Petzval would appear on an ideal intermediate image, when projecting, and image on the back of the diffuser through diffusion effect; the double-lens on the back would further project the image on the screen. A pincushion distortion therefore would appear on the Petzval surface [Fig. 4(a)]. However, when projecting to the back of the diffuser, distortion aberration would be improved from 15.79 to $6.58 \%$. The above two image planes presented the same magnified virtual images after the double-lens. It was therefore confirmed that the distortion improvement was resulted from the rough surface. Besides, the previous analyses further confirmed that the rough surface could generate a distortion-free image.

\section{Conclusions}

In conclusion, the literature mentioned that human eyes could not distinguish images with the speckle contrast dropped down to $4 \%$ and distortion rate down to $5 \% .^{11,12)}$ Nevertheless, the speckle contrast was dropped down to
$3.10 \%$ and distortion rate down to $6.58 \%$, in this experiment, by placing a diffuser at the intermediate image, and a rotating diffuser allowed the light intensity being uniformly distributed to enhance the image quality. Aiming at the requirement of a head up display projection system, the diffuser inhibiting laser speckles and enhancing the image quality of distortion aberration at the intermediate image could have the laser projection system replace the past head up displays with LED backlight. It achieves the objectives of far image distance, large projecting area, and high image quality.

\section{Acknowledgment}

This work is supported by the National Science Council of Taiwan under Contract No. NSC 101-2628-E-224-002-MY3.

\section{References}

1) L. Wang, T. Tschudi, T. Halldorsson, and P. R. Petursson: Appl. Opt. 37 (1998) 1770.

2) M. Sun and Z. Lu: Opt. Eng. 49 (2010) 024202.

3) M. Nadeem Akram, Z. Tong, G. Ouyang, X. Chen, and V. Kartashov: Appl. Opt. 49 (2010) 3297.

4) S. Jutamulia, T. Asakura, and H. Ambar: Optik 70 (1985) 52.

5) J. C. Dainty: Laser Speckle and Related Phenomena (Springer, Berlin, 1984) 2nd ed., p. 25.

6) H. Ambar, Y. Aoki, N. Takai, and T. Asakura: Appl. Phys. B 38 (1985) 71.

7) W. C. Su, C. Y. Chen, Y. F. Wang, Y. W. Chen, and S. S. Yang: J. Opt. 13 (2011) 105401.

8) S. Roelandt, Y. Meuret, G. Craggs, G. Verschaffelt, P. Janssens, and H. Thienpont: Opt. Express 20 (2012) 8770.

9) Y. Kuratomi, K. Sekiya, H. Satoh, T. Tomiyama, T. Kawakami, B. Katagiri, Y. Suzuki, and T. Uchida: J. Opt. Soc. Am. A 27 (2010) 1812.

10) J. W. Goodman: Speckle Phenomena in Optics: Theory and Applications (Ben Roberts and Co., Englewood, CO, 2006) p. 59.

11) R. Martins, V. Shaoulov, Y. Ha, and J. Rolland: Opt. Express 15 (2007) 14530.

12) Y. C. Liu and M. H. Wen: Int. J. Hum.-Comput. Stud. 61 (2004) 679. 